\title{
VAN LCOS Microdisplays: A Decade of Technological Evolution
}

\author{
Dieter Cuypers, Herbert De Smet, and André Van Calster
}

(Invited Paper)

\begin{abstract}
Microdisplays of the liquid crystals on silicon (LCOS) type have gone through a rapid evolution during the last decade. We present an overview of how vertically aligned nematic (VAN) LCOS have evolved from an attractive, but notoriously difficult and even infamous technology, to the mainstream microdisplay technology that it is today. At the same time, we highlight a number of remaining issues and concerns, and present some ideas of how to remedy them.
\end{abstract}

Index Terms-Liquid crystals on silicon (LCOS), microdisplay, vertically aligned nematic (VAN).

\section{INTRODUCTION}

M ORE than a decade ago, the concept of miniature reflective displays produced directly on top of CMOS circuitry technology started to enjoy quite a lot of interest.

Market prospects encompassing applications as diverse as projection television, small and medium venue business projectors, home cinema, simulator screens, and digital cinema were looking very fine for high-performance projectors of any kind. It was expected that the combination of two very mature technologies-liquid crystal cell manufacturing and CMOS processing - could fulfill the demands posed by these applications and create a synergy to produce very cost-effective solutions.

The idea of these liquid crystal on silicon (LCOS) devices, as they became known, had already been around for some time. A basic demonstration was presented about a decade earlier [1], however, in the following years, some fine prototypes were developed [2]-[6], and the D-ILA chip [7], a modification of the ILA device [8] turning it into an LCOS, became commercially available.

Contrary to what was expected, the development of a mature LCOS technology proved to be quite challenging and time consuming. In this paper, some aspects of this technological development will be treated. Since the scope of applications for

Manuscript received February 26, 2010; revised June 02, 2010; accepted June 04, 2010. Date of publication July 26, 2010; date of current version February 08, 2011.

D. Cuypers is with the Centre for Microsystems Technology, IMEC, B-9052 Zwijnaarde, Belgium, (e-mail: dieter.cuypers@elis.ugent.be).

H. De Smet and A. Van Calster are with the Centre for Microsystems Technology, Department of Electronics and Information Systems, Ghent University, B-9052 Zwijnaarde, Belgium (e-mail: herbert.desmet@elis.ugent.be; andre.vancalster@elis.ugent.be).

Color versions of one or more of the figures are available online at http:// ieeexplore.ieee.org.

Digital Object Identifier 10.1109/JDT.2010.2053018
LCOS devices is very broad, a restriction in terms of application area is needed to keep a concise overview. In this case, the adopted point of view is that of LCOS panels intended for projection. More explicitly, this means the assumed technology platform is that of reflective panels using nematic liquid crystals.

\section{LIQUid CRYSTAL TECHNOLOGY}

Although the early prototypes of LCOS projection light valves used either polarizer-free liquid crystal modes, such as polymer-dispersed liquid crystal (PDLC) [4], [6] and nematic curvilinear aligned phase (NCAP) [9], or used twisted nematic derived modes [3], [10], the mode of choice for LCOS devices is the vertically aligned nematic mode (VAN). This preference is based on the superior characteristics of VAN in some areas, notably contrast and brightness combined with very reasonable performance in all other areas, such as driving voltage, response speed and acceptance angle.

The vertically aligned mode requires liquid crystal mixtures with negative dielectric anisotropy. Previously, such liquid crystal types were only used in some niche applications using only a few commercially available mixtures. The advent of the microdisplays fueled interest in negative anisotropy liquid crystals, but since the expected sales volume is inevitably low, development remained relatively slow. Only when the VAN mode was also adopted by the flat-panel TV manufacturers, a larger choice of VAN mixtures became available.

The initially available mixtures actually had quite suitable properties for their application. The development of new mixtures was therefore focused on two aspects: on one hand, obtaining faster switching times and on the other hand, especially in the later years, increasing the birefringence.

Obtaining faster switching speeds in liquid crystal mixtures amounts to reducing the rotational viscosity while keeping the other physical parameters constant. In the case of VAN, with its negative anisotropy, this results in a balancing exercise between the opposing needs for polar groups placed sideways of the main molecule chain to obtain the negative birefringence while at the same time minimizing the hindrance caused by these protruding fragments to facilitate the rotation of the molecules [11].

This is achieved by fundamental research into suitable chemical compounds [12], [13], resulting in new mixture formulations. Another possibility is to add a dopant to existing liquid crystal mixtures in order to change their hydrodynamic properties without altering the birefringent and dielectric properties. One successful report uses non-planar halogenated compounds to reduce the switching time [14]. 
Increasing the birefringence is actually an indirect way to obtain faster switching cells: it allows a reduction of the cell gap, which directly affects the response speed. Producing thinner cells of course has repercussions on the manufacturing and yield of the devices, which explains the reluctance to follow this road, especially for microdisplays with a larger area.

Mixtures with higher birefringence while maintaining or slightly reducing the rotational viscosity have been developed mainly for the flat-panel TV application [13]. For microdisplays intended for projection, an additional constraint has to be taken into account. Although all projectors are equipped with UV-filters, interaction with short-wavelength blue light is the main cause of liquid crystal deterioration in microdisplays. High-birefringence mixtures tend to be more unstable under UV-irradiation than their standard counterparts. Consequently, dedicated development was undertaken to obtain highly stable, tailored mixtures for the digital cinema application [15].

Another aspect that has to be taken into account during liquid crystal mixture selection is the needed voltage to drive the cell. This comes down to the requirement that the dielectric anisotropy must be large enough. Increasing the dielectric anisotropy may however affect the quality of the vertical alignment of the liquid crystal [16]. This can be alleviated by adding small amounts of liquid crystal materials that have a positive anisotropy [16].

\section{AlignMENT TECHNOLOGY}

Contrary to the majority of the industry using nematic liquid crystals, the liquid crystal alignment technology for LCOS microdisplays does not rely on rubbed polyimide layers. This is certainly true and understandable for devices using VAN liquid crystal, for which the standard alignment layers never had proven to be a good technique. However, even LCOS devices employing classic twisted nematic liquid crystal have a tendency to use other, contact-free methods of aligning [17] because of the nature of the silicon backplane, which is quite sensitive to scratches, electrostatic discharges and the like.

In the following sections the different techniques are discussed both from a theoretical and practical viewpoint.

\section{A. Spin-On Techniques}

1) Surfactants: The classic technique to obtain a vertical alignment with suitable liquid crystals is the use of surfactants. Such molecules adhere well to electrode materials such as ITO and aluminum and align the liquid crystal molecules in the desired way by means of steric hindrance [18], [19]. The most used materials are trialkoxysilane, n,n-dimethyl n-octadecyl 3-aminopropyl tri-methoxy sylil chloride (DMOAP) and n-methyl 3-aminopropyl trimethoxysilane.

For a tilted homeotropic alignment, the treatment with DMOAP or long-chain alcohols is often combined with an obliquely deposited alignment layer [20]. The additional layer is deposited first, using the oblique evaporation technique described further on. The surface is then exposed to the coupling agent for a predetermined amount of time. Again, the long tail of the coupling agent's molecules should orient the liquid crystal homeotropically, while the underlaying layer, where the polar head of the molecule is attached, should introduce a small pretilt angle in the preferred direction.

The major problem with this technique is the reproducibility and stability. Pretilt angles are unpredictable as they are critically dependent on the exposure time and ambient conditions for the coupling agent. The surfactant layer will inevitably dissolve into the liquid crystal material thus breaking the alignment and rendering the cell useless. This technique has long time been in use in many laboratories, but is not used in any practical application for obvious reasons.

2) Polyimides: It is possible to obtain homeotropic liquid crystal alignment using dedicated polyimides (featuring alkyl side-chains), e.g the well known SE-1211 from Nissan Chemical Industries. Without further measures, these polyimides align the molecules almost perfectly vertical, which is very impractical. To achieve a certain pretilt, the layer should be rubbed. This however reintroduces the risk for damage to the silicon backplane such as scratches, contamination with foreign particles and electrostatic discharges.

One way to overcome the need for rubbing to obtain a pretilt angle is the use of protrusions or similar features in the liquid crystal layer. The orientation can then remain perfectly vertical, because the protrusions create suitable deviations in the electrical field lines when the liquid crystal is switched so that a predefined tilt is obtained. This method is best known from the larger direct-view LCD panels where it is used to create multi-domain switching, i.e., each pixel is divided into subpixels which have distinctive tilt directions. Some attempts to employ this technique for LCOS devices have been undertaken [21] with suitably smaller surface relief. The additional steps and cost to make the needed relief make this approach however less attractive.

More recently, the mixing of different aligning polyimides to introduce a controllable pretilt angle has been investigated. A planar aligning and a vertical aligning polyimide are either coated on top of each other [22] or mixed with each other before application [23] to obtain vertical alignment with a certain pretilt, depending on the relative concentration of both polyimides. Although these techniques are very promising, they still lack the maturity to make it into production. An additional disadvantage is the fact that to ensure a homogeneous azimuthal orientation, a weak rubbing step is still required.

3) Photo-Alignment: The photo-alignment technique has for quite some time been promoted as an easy, contact-free alternative for the conventional alignment layers. In the original report by Gibbons [24], polyimide films doped with photo-sensitive azo-dye compounds are used. Later on mainly poly-vinyl-cinnamate and its derivatives have been used [25]-[27], but also polyimides [28] and other reactive polymers [29]. Alignment of liquid crystal molecules is for most polymers triggered by a process of photodegradation. Linearly polarized ultraviolet light breaks bonds in the polymer in a preferential direction and this anisotropy in the aligning layer is reproduced in the liquid crystal orientation. For vertical alignment, it is possible to use non-polarized obliquely incident light [30].

Despite the clear advantages of the technique, it has never really caught on in production environments. Reasons are the poor thermal stability and the sensitivity to high energy photons 
which can alter the alignment characteristics during the lifetime of the devices [31]. Another reason is the generally weak anchoring that is obtained with the photo-alignment materials.

4) Polymer-Stabilized Alignment: One of the latest developments is the so-called polymer-sustained alignment for VAN liquid crystal [32]-[34]. For this, reactive mesogens are mixed in the liquid crystal material at a low concentration so that no polymer network will be formed in the bulk of the liquid crystal layer. With the appropriate ultraviolet curing dosage and bias voltage, the liquid crystal directors can be fixed (sustained by the polymer surface) at a certain small pretilt angle. This technique is very promising as it eliminates most problems and drawbacks of the other techniques without additional cost and even seems to enable higher switching times. Since its development started only very recently, industrial application is still ramping up.

\section{B. Oblique Particle Beam Techniques}

A completely different approach to aligning liquid crystals, but almost as old as the polyimide techniques, is the use of beams of oblique particles. Most widespread is the deposition of the alignment material (usually an inorganic substance) under oblique conditions, but also oblique etching of previously deposited alignment material can be performed. The advantage of the oblique particle beam techniques is foremost the inherent contact-less nature of forming the alignment layer, posing no risk of damaging the substrate either mechanically or by electrostatic discharge. As a bonus, the deposited layers are usually very inert, so less influence on the optical and dielectric behavior of the device is to be expected and a superior stability removes all concerns about lifetime and reliability of the alignment system.

1) Oblique Evaporation of Inorganic Alignment Layers: The classic technique (producing homogeneous alignment) consists of the static evaporation at nearly grazing incidence of an inorganic substance [35]. The preferred material is siliconoxide, $\mathrm{SiO}_{x}$, with $x$ frequently being 1 or at least less than 2 . Occasionally some reports on aluminumoxide can be found [36]. It is interesting to note that successful attempts to use indium-tin-oxide (ITO) have never been reported, despite the attractiveness of the combined functionality of electrode and alignment layer.

To overcome some of the drawbacks of the initial technique and produce different types of alignment, a number of variants have been proposed. These ranged from simple geometrical variations [37], [38], over the introduction of louvers [39] or apertures [37], [40] to advanced configurations using double depositions [41]-[44].

A particularly versatile and reliable technique is the rotation deposition technique [45]. The substrate is mounted obliquely with respect to the evaporation source, but can be rotated around its center. The rotation speed can be varied continuously depending on the orientation of the substrate. By choosing, e.g., a sinusoidal relation between the angular speed and the orientation, the azimuthal distribution of the deposition can be changed. Depending on this distribution, a wide range of pretilt angles is obtained. The complexity of the equipment needed to provide the angular motion and the difficulty to coat several substrates at a time have prevented widespread use of this technique.

The choice for $\mathrm{SiO}_{x}$ has however some drawbacks. First of all, $\mathrm{SiO}_{x}$ is not stoichiometrically stable, so every layer will have a certain tendency to further oxidize towards $\mathrm{SiO}_{2}$. For the same reason it can be difficult to obtain exactly the same material in-between different production batches. Silicondioxide eliminates most of these problems. It is very stable stoichiometrically and inert under all circumstances encountered in display use, e.g., under ultraviolet radiation.

The introduction of silicondioxide in combination with simple, single deposition methods thus marked the start of the production of reliable VAN microdisplays [10], [46], [47]. As of today, virtually all VAN LCOS production lines rely on these evaporation methods for the alignment layers.

2) Oblique Sputtering of Alignment Layers: Because the oblique evaporation process was considered a not so efficient process, and the process equipment does not lend itself so easily to adoption in a high volume production environment, attempts have been made to replace the evaporation by the sputter deposition technique, which would allow faster deposition cycles and larger substrate sizes.

It is obvious that the nature of the sputtering deposition method is not in good agreement with the need for a highly directional material beam. The bombardment of the target with heavy argon ions effectively results in target material atoms hitting the substrate at every possible angle, evidenced by the highly conformal nature of sputtered coatings.

To obtain anisotropy in the deposited layer some rely on changing incidence angles generated by a substrate passing under the target [48]. Another well-known system starts from a vertical in-line sputtering machine which is adapted to allow oblique deposition [49]. The direction-less target material beam is transformed into a directional one by placing sets of louvers between the target and the substrates. Oblique incidence is obtained by mounting the rectangular target on wedges, so that it is not parallel with the substrates anymore.

The produced alignment films are of course highly uniform, also on large substrates, and this is also reflected in the uniformity of the vertical alignment. However, it has proven to be very difficult to obtain larger pretilt angles with the sputtering technique. Consequently, only few assembly lines make use of the sputtering technique today.

3) Ion Beam Writing and Plasma Treatments: Introducing alignment by treating a layer with an ion flux was demonstrated a while ago [50]. The version that has become most well known has been presented by the display group of IBM [51], using a lower energy flux than in the original.

The proposed solution uses a diamond-like-carbon hard-coat, $3-15 \mathrm{~nm}$ thick deposited via chemical vapor deposition or sputtering. The alignment is obtained by bombarding the layer with argon ions under an oblique incidence angle. Homogeneous alignment with a controllable pretilt angle of $0^{\circ}$ to $10^{\circ}$ (from horizontal) and strong anchoring is achieved. Homeotropic alignment was reported a few years later [52], using fluorinated diamond-like-carbon. Again, controllable pretilt angles from $71^{\circ}$ to $89.8^{\circ}$ could be produced. 
The explanation offered for the mechanism of alignment is based on a random network model of the carbon atom arrangement in the film. Order is then induced by exposure to the ion beam because unfavorably oriented rings of atoms are selectively destroyed. The planes of the remaining rings are predominantly parallel to the direction of the ion beam and will thus induce alignment of the liquid crystal molecules[53]. For the homeotropic alignment the mechanism is less clear, although the fluorohydrocarbons are believed to contribute to the vertical alignment by means of their extremely low surface tension [52].

Similar results have been obtained using argon plasma irradiation of different base materials, both organic and inorganic and for homogeneous and homeotropic alignment [54]-[56].

The advantage of these methods lies in their flexibility and reliability; they probably offer the highest versatility of all the contact-less alignment methods. The cost and relative complexity of the equipment needed for the deposition and treatment of these films is of course the main drawback of the process. Even compared to the evaporation methods, which already require a serious investment, the costs are as yet prohibitively high .

\section{Alignment Mechanism}

Some efforts have been put into a better understanding of the physics behind the alignment of liquid crystal molecules to the obliquely deposited layers. Although a general consensus about the nature of the alignment action seems to have been reached, there are still a number of experimental results that seem difficult to fit into the model.

1) Layer Morphology: For a long time, the alignment action has been attributed to the peculiar morphology associated with obliquely deposited layers.

Films deposited with an obliquely incident vapor stream will in general exhibit a columnar morphology. The axis of the columns will be inclined toward the impinging vapor stream, with the actual angle somewhere in-between the substrate normal and the incidence angle, depending on the deposition circumstances. The columns will also bundle themselves in rows, perpendicular to the plane of incidence, with the columnar separation being larger between the respective bundles than inside the bundles. The cross-section of the columns is generally elliptical. For crystalline materials, a specific texture is also observed, i.e., the orientation of the main axes of the crystal are dependent on the incidence angle and the deposition parameters [57].

Different theoretical models have been proposed to explain the observed features of obliquely deposited films. The inclination of the columns has been described with the discretized continuum model [58], self-shadowing [58], conservation of parallel momentum [59], [60] and angle dependent growth [57], [61]. Self-shadowing [62], [63] and conservation of parallel momentum [64] can also be used for the bundling phenomenon. The shape of the cross-section of the columns can again be predicted by the conservation model [65], while the formation of texture is described by surface diffusion effects [66].

Although the formation of the typical morphology of these layers is thus well understood, it has been very difficult to explain the alignment behavior of the liquid crystal solely based on the characteristics of the layer. There is very little correlation between the properties of the layer and the resulting alignment.
It is now believed that the morphology of the layer is a second order effect which contributes only to the variations in pretilt angle [67].

2) Anchoring: Contrary to the anchoring on polyimide surfaces, which is believed to be caused by coupling of $\pi$ electrons between polyimide and liquid crystal molecules [68], the anchoring on inorganic surfaces is attributed to induced dipoledipole interactions [69]. In the absence of other interactions, such as steric hindrance, intrinsic dipoles or geometrical effects, which may be dominant, the Van der Waals force between the liquid crystal molecules and the alignment layer molecules is considered the determining factor for the type of alignment.

This statement is inferred from the observation that liquid crystals with positive anisotropy align homogeneously while negative anisotropy mixtures align homeotropically on otherwise identical inorganic surfaces. Excluding the above mentioned other types of interaction, the anchoring force between LC and surface must be caused by induced dipole to dipole interactions. This induced dipole interaction is based on attractive Van der Waals forces which can be written as a potential of the form $-\lambda / r^{6}$, where the constant $\lambda$ is proportional to the product of the polarizabilities of the materials. The polarizability of the aligning layer is obviously constant, but the LC has two polarizabilities, corresponding with the anisotropy in dielectric permittivity. This is readily apparent from the Clausius-Mossotti equation, relating the molecular polarizability $\alpha$ of a non-polar medium to the dielectric permittivity. This directly leads to the conclusion that materials with positive anisotropy will align homogeneously, while negative anisotropy means a preference for homeotropic alignment [70].

To explain the variation of the pretilt angle with the deposition parameters, the layer morphology has to be taken into account. This is accomplished via the introduction of an order-electricity term in the anchoring energy. This introduction is presented as a logical extension to the classical theory of Barbero et al. [71] regarding surface orientation.

In the paper of Barbero, it is shown that in a nematic liquid crystal, a net polarization can exist if a spatial gradient in the order parameter $S$ is present while the director $\vec{n}$ remains constant. This polarization is similar, in nature and magnitude, to the flexoelectric polarization that occurs under the opposite conditions, a non-uniform director profile with constant order parameter [72]. This order polarization term must be taken into account for the internal energy of the entire system, hence it influences the equilibrium configuration of that system.

Extrapolating the conclusions of Barbero and applying them to interfaces of liquid crystal with a solid alignment layer, it can be deemed plausible that order polarization plays its role here as well. Depending on the layer morphology, the order polarization term will vary and so will the pretilt angle which minimizes the system energy. The theory then predicts that for a liquid crystal with positive anisotropy the pretilt angle should always be less than $35.3^{\circ}$ (from horizontal), whereas for negative anisotropy, the complementary angle should be used, so pretilt is expected to be larger than $54.7^{\circ}$. This seems to be in line with a number of measurements, although for example Hiroshima [45] reports pretilt angles for negative anisotropy liquid crystal ranging from $30^{\circ}$ to $90^{\circ}$, which is one case where the model clearly does not hold. 


\section{Device Performance Parameters}

\section{A. Contrast}

One of the initial attractive features of the VAN mode is that it has an inherent high contrast, virtually independent of wavelength or cell gap. This is due to the almost vertical orientation of the liquid crystal molecules in the black state, which keeps residual birefringence to a minimum and results in a contrast ratio that is close to the contrast ratio of the crossed polarizers themselves.

Consequently, the initial concern was to obtain a uniform alignment with a very small pretilt angle ( $1^{\circ}$ to $2^{\circ}$ away from vertical) to preserve this contrast. Of course, this line of reasoning only applies to the on-axis modulation of the light. Offaxis light rays are not so well modulated. This is partly due to the incorrect retardance incurred in the liquid crystal stack, but the contrast ratio is also strongly affected by the skew ray depolarization that occurs when using McNeille polarizing beamsplitters [73]. The skew ray depolarization can be greatly remedied by inserting a quarter-wave plate in the optical path. In later years, the adoption of wire-grid polarizers in optical engines [74], [75] made this issue less of a concern since this type of polarizers suffers much less of skew ray depolarization [76].

The approach outlined above, i.e., aiming for very small pretilt angles has a number of drawbacks, most notably a slow switching speed caused by a delay before the molecules start to tilt and disclination lines that can occur through fringing fields [77]. A slightly higher (i.e., further away from vertical) pretilt angle alleviates this greatly, but of course would reduce contrast if applied without further measures [78].

To allow the increase of the pretilt angle to within the range of $2^{\circ}$ to $10^{\circ}$ away from vertical, compensation foils are used. A first compensation aims at restoring the on-axis contrast, which means countering the in-plane retardance caused by the slightly tilted molecules. This requires a negative a-plate with a suitable retardance. The out-of-plane retardance, which manifests itself as a slightly elliptical polarization in the off-axis rays, should be compensated with a negative c-plate [79]. Fabrication of negative c-plates is not so easy, so some workarounds have been proposed, such as replacing the negative c-plate with two crossed positive a-plates, which give similar results [80].

Fully compensated high-end panels can nowadays easily achieve a 5000:1 contrast ratio for an illumination system with an f-number of 2.0.

\section{B. Response Speed}

The concern for a suitably high response speed is driven by a number of considerations. First, there is of course the basic requirement that the switching speed be high enough to keep pace with the frame rate of standard video sources. Even with the early VAN liquid crystal mixtures, this requirement was easily met as soon as the cell gap was sufficiently thin, i.e., around $3 \mu \mathrm{m}$.

A second consideration, and one that is far more challenging, is the desire to have an LCOS that can operate in color sequential mode. This would allow single chip optical engines, reducing the cost significantly. It is possible to obtain single panel operation by using color filters on the panel, either conventional [81] or the more advanced holographic ones [82], but this inevitably leads to either resolution loss or larger panels and is therefore not favored.

The potential for color sequential operation of VAN cells has been reported a number of times [83], [84], but a real breakthrough has never occurred. The required speed is obtained by reducing other performance factors (notably optical efficiency and sometimes contrast), and as a consequence these panels are not used for high quality applications. Color sequential VAN microdisplays can nowadays be found in mobile applications such as pico-projectors. High-end LCOS panels nowadays can have response times (i.e., $t_{\text {on }}+t_{\text {off }}$ ) down to $5 \mathrm{~ms}$.

Lastly, a higher response speed is beneficial to improve the perceived image quality of sample-and-hold type displays, such as liquid crystal devices. These image quality issues are mostly related to motion artifacts [85]. Blurring of moving objects is perceived because the eye tracks such objects smoothly, whereas sample and hold displays feature sudden transitions at the pace of the frame-rate [86]. Motion blur has been addressed quite extensively in more recent years by flat-panel TV makers. Although not completely adequate, the main characteristic used for evaluating liquid crystal performance for moving imagery has settled upon the Motion Picture Response Time (MPRT) [87]. Most of the observations made also pertain to microdisplays, although some of the solutions (e.g., overdrive techniques) are seldom used. The benefit of higher response speed in this case is that it can enable solutions like black frame insertion or increased frame-rates.

\section{Miscellaneous}

\section{A. Contamination}

A point of concern for the use of evaporation of inorganic alignment layers is the highly hydrophilic nature of the $\mathrm{SiO}_{2}$ and its tendency to adsorb hydrocarbon fractions present in the environment. These fractions are disturbing the anchoring mechanism described above as they definitely alter the Van der Waals forces between aligning layer and liquid crystal. If excessive, the alignment may effectively change from homeotropic to homogeneous [88]. Under normal assembly conditions, the alignment layers are never exposed long enough to trigger this event. However, a similar contamination can easily happen during the application of the glue to bond the panels together. Uncured glue is then directly in contact with the alignment layer and can readily be absorbed. Not fully cured glue may even bleed small hydrocarbons during the lifetime of the device and thus affect reliability. The solution was found in a careful selection of the glue type and a well-controlled curing process [88].

\section{B. Vcom Drift}

LCOS lightvalves require an offset voltage superimposed on the driving waveform to obtain a flicker-free output. The need for this so-called Vcom offset voltage stems from the presence of an internal voltage in the cell itself, the origin of which is still debated. Interest in the mechanism of the generation of the Vcom voltage is driven by the fact that it is a time dependent factor and thus cannot be easily compensated for, which in turn makes it a threat for the flawless operation of the lightvalve [89]. 
Originally, the source of the Vcom voltage was thought to be the difference in work-function of the two electrodes [10]. One of the advantages cited for the introduction of diamond-likecarbon alignment layers was actually that these slightly conductive layers would constitute a pair of electrodes with equal work-functions, thus eliminating the Vcom voltage [90]. A dependence on time is however very hard to explain with this model.

For devices with polyimide alignment layers, the battery model has long been in favor, where the electrode-polyimide - liquid crystal stack is considered a galvanic cell and the Vcom voltage a manifestation of the electromotive force [91]. Time dependent changes in the Vcom voltage consequently are attributed to depletion of the battery.

The major flaw in this theory is the fact that it not able to explain the reversibility of the Vcom drift that is observed in most cells. Moreover, cells with inorganic alignment layers exhibit a very similar Vcom drift behavior, but seem not to fit within the explanation offered by this model [92].

As an alternative model, we propose to look at the alignment layer as a medium that is somewhat penetrable for small charged particles (e.g., electrons). It thus contains a number of trapped charges, but also allows the transfer of charges. At the interface with the liquid crystal an electrochemical exchange reaction takes place which results in a small equilibrium potential over the interface. The sum of the potentials over both alignment layer interfaces thus constitutes the observed internal voltage. As both interfaces are not identical, the equilibrium conditions for both in varying circumstances (external voltage, temperature, etc) need not be the same, thus offering an explanation for both the time dependence and the reversibility.

The Vcom drift effect in todays devices is minimal, but still needs attention when continuous operation over longer periods is foreseen.

\section{CONCLUSION}

The most important highlights in the technological development of VAN microdisplays have been treated.

\section{REFERENCES}

[1] I. Underwood, "A NMOS addressed liquid crystal spatial light modulator," Ph.D. dissertation, Univ. of Edinburg, Edinburgh, U.K., 1987.

[2] E. Colgan and M. Uda, "On-chip metallization layers for reflective light valves," IBM J. Res. Develop., no. 42, pp. 339-345, 1998.

[3] R. L. Melcher, P. M. Alt, D. B. Dove, T. M. Cipolla, E. G. Colgan, F. E. Doany, K. Enami, K. C. Ho, I. Lovas, C. Narayan, R. S. Olyha, C. G. Powell, A. E. Rosenbluth, J. L. Sanford, E. S. Schlig, R. N. Singh, T. Tomooka, M. Uda, and K. H. Yang, "Design and fabrication of a prototype projection data monitor with high information content," IBM J. Res. Develop., vol. 42, no. 3-4, pp. 321-336, 1998.

[4] P. Cacharelis, U. Kim, J. Frazee, P. Moore, K. Brown, R. Luttrell, P. Rentein, and R. Flack, "An 0.8- $\mu \mathrm{m}$ EEPROM technology modified for a reflective PDLC light-valve application," in SID Int. Symp. Dig. Tech. Papers, Boston, MA, May 1997, vol. XXVIII, pp. 289-292.

[5] F. Sato and Y. Yagi, "High resolution and bright LCD projector with reflective LCD panels," in SID Int. Symp. Dig. Tech. Papers, Boston, MA, May 1997, vol. XXVIII, pp. 997-1000.

[6] T. Nagata, I. Takemoto, T. Miyazawa, A. Asano, K. Yanagawa, S. Nakamura, H. Nakagawa, K. Saitou, N. Okabe, K. Matsumoto, and A. Iguchi, "Silicon chip based reflective PDLC light valve for projection display," in SID Int. Symp. Dig. Tech. Papers, Anaheim, CA, May 1998, vol. XXIX.
[7] H. Kurogane, K. Doi, T. Nishihata, A. Honma, M. Furuya, S. Nakagaki, and I. Takanashi, "Reflective AMLCD for projection displays: D-ILA," in SID Int. Symp. Dig. Tech. Papers, Anaheim, CA, May 1998, vol. XXIX.

[8] R. Sterling and W. Bleha, "Electronic cinema using ILA projector technology," in SID Int. Symp. Dig. Tech. Papers, San Jose, CA, May 1999, vol. XXX.

[9] Y. Nagae, K. Ando, A. Asano, L. Takemoto, J. Havens, P. Jones, D. Reddy, and A. Tomita, "Compact liquid-crystal projectors with high optical efficiency," in SID Int. Symp. Dig. Tech. Papers, Orlando, FL, May 1995, vol. XXVI, pp. 223-226.

[10] M. Lu and K. Yang, "Reflective nematic LC devices for LCOS applications," in SID Int. Symp. Dig. Tech. Papers, Long Beach, CA, May 2000, vol. XXXI.

[11] M. Hird, J. W. Goodby, and K. J. Toyne, "Nematic materials with negative dielectric anisotropy for display applications," in Liquid Crystal Materials, Devices, and Flat Panel Displays, R. Shashidhar, Ed. San Jose, CA: IS\&T, 2000, vol. 3955, pp. 15-23.

[12] M. Klasen-Memmer, M. Bremer, and K. Tarumi, "Advanced liquid crystal materials with negative dielectric anisotropy for monitor and TV applications," in Proc. 9th Int. Display Workshops, Hiroshima, Japan, Dec. 2002, vol. XXVII, pp. 93-95.

[13] D. Pauluth and K. Tarumi, "Optimization of liquid crystals for television," J. Soc. for Inf. Display, vol. 13, no. 8, pp. 693-702, 2005.

[14] P. Kilickiran, A. Masutani, N. Hollfelder, G. Nelles, A. Yasuda, A. Tadeusiak, and G. Sandford, "Halogenated non-planar dopants for fast response liquid crystals," in SID Int. Symp. Dig. Tech. Papers, 2007, vol. XXXVIII, pp. 999-1002.

[15] P. Kula, A. Spadlo, J. Dziaduszek, M. Filipowicz, R. Dabrowski, J. Czub, and S. Urban, "Mesomorphic, dielectric, and optical properties of fluorosubstituted biphenyls, terphenyls, and quaterphenyls," OptoElectron. Rev., vol. 16, no. 4, pp. 379-385, 2008.

[16] C. Chen, P. J. Bos, J. Kim, Q. Li, and J. E. Anderson, "Improved liquid crystals for vertical alignment applications," J. Appl. Phys., vol. 99, no. 12, pp. 123 523-123 529, 2006.

[17] P. Chaudhari, J. A. Lacey, S.-C. A. Lien, and J. L. Speidell, "Atomic beam alignment of liquid crystals," Jpn. J. Appl. Phys., vol. 37, no. 1, pp. L55-L56, 1998.

[18] F. Kahn, "Orientation of liquid crystals by surface coupling agents," Appl. Phys. Lett., vol. 22, p. 386, 1973.

[19] S. Matsumoto, K. Kawamoto, and N. Kaneko, "Surface-induced molecular orientation of liquid crystals by carboxylatochromium complexes," Appl. Phys. Lett., vol. 27, p. 268, 1975.

[20] A. Masano, K. Toshio, S. Shigeo, N. Hiroyuki, S. Masanobu, S. Tadayuki, and , Victor Company, Japan, "Liquid crystal display device and method of producing the same," (France, Germany and U.K.) European Patent 0541388, May 12, 1991.

[21] M. Gardner, S. Day, and F. Fernandez, L. Mazuray, P. Rogers, and R. Wartmann, Eds., "Liquid crystal VAN tilt bias by surface relief patterning," in Proc. SPIE, 2004, vol. 5249, pp. 729-739.

[22] K. Zhang, N. Liu, R. J. Twieg, and P. Bos, "Pretilt study of double layer alignment film," Liquid Crystals, vol. 35, pp. 1191-1197, 2008.

[23] K. E. Vaughn, M. Sousa, D. Kang, and C. Rosenblatt, "Continuous control of liquid crystal pretilt angle from homeotropic to planar," Appl. Phys. Lett., vol. 90, no. 19, p. 194102, 2007.

[24] W. Gibbons, P. Shannon, S. Sun, and B. Swetlin, "Surface-mediated alignment of nematic liquid crystal with polarized laser light," Nature, vol. 351 , p. $49,1991$.

[25] M. Schadt, M. Schmitt, V. Kizinkov, and V. Chigrinov, "Surface-induced parallel alignment of liquid crystals by linearly polymerized photopolymers," Jpn. J. Appl. Phys., vol. 31, pt. 1, p. 2155, 1992.

[26] Y. Iimura, T. Saitoh, S. Kobayashi, and T. Hashimoto, "Liquid crystal alignment on photopolymer surfaces exposed by linearly polarized UV light," J. Photopolymer. Sci. Technol., vol. 8, no. 2, pp. 257-262, 1995.

[27] Y. Iimura, H. Akiyama, X. Li, and S. Kobayashi, "Photo-alignment control of LC and its applications to LCD fabrication," in Liquid Crystal Materials, Devices and Apllications VI, R. Shashidhar, Ed. San Jose, CA: IS\&T, 1998, vol. 3297, pp. 8-17.

[28] J. West, M. Nishikawa, and Y. Reznikov, "Generation of high pretilt in photo-aligned polyimides," in Liquid Crystal Materials, Devices and Apllications VII, R. Shashidhar, Ed. San Jose, CA: IS\&T, 1999, vol. 3635, pp. 16-22.

[29] P. Jackson, R. Karapinar, M. Neill, P. Hindmarsh, G. Owen, and S. Kelly, "Alignment models for coumarin-containing polymers for liquid crystal displays," in Liquid Crystal Materials, Devices and Applications VII, R. Shashidhar, Ed. San Jose, CA: IS\&T, 1999, vol. 3635, pp. 38-47. 
[30] V. Konovalov, V. Chigrinov, H. S. Kwok, H. Takada, and H. Takatsu, "Photoaligned vertical aligned nematic mode in liquid crystals," Jpn. J. Appl. Phys., vol. 43, pp. 261-266, 2004.

[31] K. Yang, K. Tajima, A. Takenaka, and H. Takano, "Charge trapping properties of UV-exposed polyimide films for the alignment of liquid crystals," Jpn. J. Appl. Phys., vol. 35, pt. 2, p. L561, 1996.

[32] K. Hanaoka, Y. Nakanishi, Y. Inoue, S. Tanuma, Y. Koike, and K. Okamoto, "A new MVA-LCD by polymer sustained alignment technology," in SID Int. Symp. Dig. Tech. Papers, 2004, vol. XXXV, pp. $1200-1203$.

[33] A. Götz, M. Klasen-Memmer, M. Bremer, A. Taugerbeck, K. Tarumi, and D. Pauluth, "Advanced LC materials for fast switching display modes," in Proc. 15th Int. Display Workshops, Niigata, Japan, Dec. 2008, pp. 1527-1530.

[34] C.-Y. Huang, W.-Y. Jhuang, C.-T. Hsieh, and C.-H. Lin, "Switching of polymer-stabilized vertical alignment liquid crystal Pi cell-curing voltage and driving scheme effects," Jpn. J. Appl. Phys., vol. 48, p. 020210, 2009

[35] J. Janning, "Thin film surface orientation for liquid crystals," Appl. Phys. Lett., vol. 21, no. 4, p. 173, 1972.

[36] G. Hauck, "Liquid crystal alignment on obliquely deposited $\mathrm{Al}_{2} \mathrm{O}_{3}$ surfaces," Physica Status Solidi, vol. 70, no. 1, pp. K43-K46, 1982.

[37] T. Terasaka, K. Arai, K. Asano, and S. Nishi, Konishiroku Photo Industries, Kanagawa, Japan, "Method for manufacturing the substrate for liquid crystal display," U.S. Patent 4897 290, Jan. 30, 1990.

[38] N. Kato, R. Sekura, and T. Iwaki, Seiko Instruments Inc., Chiba, Japan, "Method of introducing slightly titlting homeotropic orientation into liquid crystal, liquid crystal electro-optical device and liquid crystal light valve," U.S. Patent 5745205 , Apr 28, 1998.

[39] M. Oue, O. Asai, K. Iwasaki, and H. Kawakami, Hitachi Ltd., Tokyo, Japan, "Method of forming alignment film for liquid crystal display cell," U.S. Patent 4043647 , Aug. 23, 1977.

[40] K. Nobuyoshi, Nissan Motor Co. Ltd., Kanagawa, Japan, "Manufacture of liquid crystal display element," Japan Patent 57084 428, May 26, 1982.

[41] A. Sawada, Citizen Watch Co. Ltd, Tokyo, Japan, "Orientation treatment of liquid crystal cell substrate," Japan Patent 56099318 , Oct. 28, 1981.

[42] Citizen Watch Company Ltd., "Alignment control of liquid crystal molecules," Patent of the United Kingdom of Great Britain, patent 1603905, Dec. 2, 1981.

[43] K. Suganuma, Citizen Watch Co. Ltd, Tokyo, Japan, "Orientation treatment method of liquid crystal cell substrate," Japan Patent 55006 306, Jan. 17, 1980.

[44] H. Vithana, Y. Yung, S. Jamal, R. Herke, P. Bos, and D. Johnson, "A well-controlled tilted-homeotropic alignment method and a vertically aligned four-domain LCD fabricated by this technique," in SID Int. Symp. Dig. Tech. Papers, Orlando, FL, May 1995, vol. XXVI, pp. 873-875.

[45] K. Hiroshima, "Controlled high-tilt-angle nematic alignment compatible with glass frit sealing," Jpn. J. Appl. Phys., vol. 21, no. 12, pp. L761-L763, 1982

[46] H. Ishino and T. Inoue, "QUALIA-004 Full high-definition home theater projector using silicon crystal reflective display (SXRD) technology," in Proc. 11th Int. Display Workshops, Niigata, Japan, Dec. 2004, pp. 1687-1688.

[47] A. Van Calster and D. Cuypers, "Reflective vertically aligned nematic liquid crystal microdisplays for projection applications," Projection Displays 2000: Sixth in a Series, vol. 3954, pp. 112-119, 2000.

[48] J. Duchene, Commissariat Energie Atomique, Grenoble, France, "Device for depositing an orientation layer of a liquid crystal cell," European Patent 0184487, 1986.

[49] R. Bürkle and T. Kallfass, "Process and apparatus for applying orienting layers to a substrate for alignment of liquid crystal molecules," U.S. Patent 5658 439, Aug. 19, 1997.

[50] M. J. Little, H. L. Garvin, and Y.-S. Lee, "Means and method for inducing uniform parallel alignment of liquid crystal material in a liquid crystal cell," U.S. Patent 4153 529, May 8, 1979.

[51] Y. Nakagawa, Y. Kato, Y. Saitoh, K. Sakai, H. Satoh, K. Wako, and S. Odahara, "Novel LC alignment method using diamond like carbon film and ion beam alignment," in SID Int. Symp. Dig. Tech. Papers, San Jose, CA, Jun. 2001, vol. XXXII.

[52] H. J. Ahn, S. J. Rho, K. C. Kim, J. B. Kim, B. H. Hwang, C. J. Park, and H. K. Baik, "Ion-beam induced liquid crystal alignment on diamondlike carbon and fluorinated diamond-like carbon thin films," Jpn. J. Appl. Phys., vol. 44, pp. 4092-4097, 2005.
[53] P. Chaudhari, J. Lacey, J. Doyle, E. Galligan, A. Lien, A. Callegari, G. Hougham, N. Lang, P. S. Andry, R. John, K.-H. Yang, M. Lu, C. Cai, J. Speidell, S. Purushothaman, J. Ritsko, M. Samant, J. Stöhr, Y. Nakagawa, Y. Katoh, Y. Saitoh, K. Sakai, H. Satoh, S. Odahara, H. Nakano, J. Nakagaki, and Y. Shiota, "Atomic-beam alignment of inorganic materials for liquid-crystal displays," Nature, vol. 411, pp. 56-59, 2001

[54] O. Yaroshchuk, R. Kravchuk, A. Dobrovolsky, L. Qiu, and O. Lavrentovich, "Planar and tilted uniform alignment of liquid crystals by plasma treated substrates," Liquid Crystal Commun., vol. 31, no. 6, pp. 859-869, 2004.

[55] O. V. Yaroshchuk, R. M. Kravchuk, A. M. Dobrovolsky, P. C. Liu, and C. D. Lee, "Plasma beam alignment for the large-area substrates: Equipment and process," J. Soc. Inf. Display, vol. 13, no. 4, pp. 289-294, 2005

[56] O. Yarushchuk, R. Kravchuk, S. Pogulyai, and S. Tsoilko, "Atmospheric plasma tool and process for tuning pretilt angle in the VAN cells," in Proc. 9th Int. Display Res. Conf., Sep. 2009, pp. 146-149.

[57] L. Abelmann and C. Lodder, "Oblique evaporation and surface diffusion," Thin Solid Films, vol. 305, pp. 1-21, 1994.

[58] A. Dirks and H. Leamy, "Columnar microstructure in vapour-deposited films," Thin Solid Films, vol. 47, pp. 219-233, 1977.

[59] K. Hara, "Anomalous magnetic anisotropy of thin films evaporated at oblique incidence," J. Science, no. 34, pp. 139-163, 1970.

[60] K. Hara, M. Kamiya, T. Hashimoto, K. Okamoto, and H. Fujiwara, "Oblique incidence anisotropy of the iron films evaporated at low substrate temperature," J. Magnet. Magn. Mater., vol. 73, pp. 161-166, 1988.

[61] S. Lichter and J. Chen, "Model for columnar microstructure of thin solid films," Phys. Rev. Lett., vol. 56, no. 13, pp. 1396-1399, 1986.

[62] H. Leamy, G. Gilmer, and A. Dirks, The Microstructure of Vapour De posited Thin Films. Amsterdam, Netherlands: North-Holland, 1980, vol. 6, Current topics in materials science, pp. 309-344.

[63] H. Leamy and A. Dirks, "The microstructure of amorphous rare earth/ transition-metal thin films," J. Phys., vol. D10, pp. L95-L98, 1977.

[64] H. Fujiwara, K. Hara, M. Kamiya, T. Hashimoto, and K. Okamoto, "Columnar growth in evaporated iron films," J. Magnet. Magn. Mater., vol. 35, pp. 296-298, 1983.

[65] J. van de Waterbeemd and G. Oosterhout, "Effect of the mobility of metal atoms on the structure of thin films deposited at oblique incidence," Philips Res. Rep., vol. 22, pp. 375-387, 1967.

[66] A. van der Drift, "Evolutionary selection, A principle governing growth orientation in vapour-deposited layers," Philips Res. Rep., vol. 22, pp. 267-288, 1967.

[67] M. Lu, K. H. Yang, T. Nakasogi, and S. J. Chey, "Homeotropic alignment by single oblique evaporation of $\mathrm{SiO} 2$ and its application to high resolution microdisplays," in SID Int. Symp. Dig. Tech. Papers, Long Beach, CA, May 2000, vol. XXXI, pp. 446-449.

[68] M. G. Samant, J. Stöhr, H. R. Brown, T. P. Russell, J. M. Sands, and S. K. Kumar, "NEXAFS studies on the surface orientation of buffed polyimides," Macromolecules, vol. 29, no. 26, pp. 8334-8342, 1996.

[69] M. Lu, K. Yang, and J. Chey, "Homeotropic alignment of liquid crystals by single evaporation of $\mathrm{SiO}_{2}$," in Proc. 6th Int. Display Workshops, Sendai, Japan, Dec. 1999, pp. 121-124.

[70] M. Lu, "Liquid crystal orientation induced by Van der Waals interaction," Jpn. J. Appl. Phys., vol. 43, no. 12, pp. 8156-8160, 2004.

[71] G. Barbero, I. Dozov, J. Palierne, and G. Durand, "Order electricity and surface orientation in nematic liquid crystals," Phys. Rev. Lett., vol. 56, no. 19 , pp. 2056-2059, 1986.

[72] R. Meyer, "Piezoelectric effects in liquid crystals," Phys. Rev. Lett., vol. 22, p. 319, 1969.

[73] A. Rosenbluth, D. Dove, and F. Doany, "Contrast losses in projection displays from depolarization by tilted beam splitter coatings," in Proc. 17th Int. Display Res. Conf., 1997, pp. 226-229.

[74] A. Kurtz, B. Silverstein, and J. Cobb, "Digital cinema projection with R-LCOS displays," in SID Int. Symp. Dig. Tech. Papers, Seattle, WA, May 2004, vol. XXXV, pp. 166-169.

[75] E. Gardner and D. Hansen, "An image quality wire-grid polarizing beam splitter," in SID Int. Symp. Dig. Tech. Papers, Baltimore, MD, May 2003, vol. XXXIV, pp. 62-63.

[76] D. Hansen, E. Gardner, R. Perkins, M. Lines, and A. Robbins, "The display applications and physics of the ProFlux wire grid polarizer," in SID Int. Symp. Dig. Tech. Papers, Boston, MA, May 2002, vol. XXXIII, pp. 730-733.

[77] D. Cuypers, H. De Smet, and A. Van Calster, "Fringe field effects in microdisplays," in SID Int. Symp. Dig. Tech. Papers, Boston, MA, Dec. 2005, vol. XXXVI, pp. 1298-1301. 
[78] X. Quintana, M. Geday, B. Cerrolaza, D. Pérez-Medialdea, and J. Otón, "Measurement of pretilt angle and cell gap in reflective vertically aligned nematics," in Proc. 6th IEEE Spanish Conf. on Electron Devices, Madrid, Spain, Feb. 2007, pp. 258-261.

[79] M. Duelli, D. M. Shemo, K. D. Hendrix, A. Ledeur, and K. L. Tan, "High performance contrast enhancing films for VAN-mode LCoS panels," in SID Int. Symp. Dig. Tech. Papers, May 2005, vol. XXXVI, pp. 892-895.

[80] V. Sergan, P. Bos, and G. Sharp, "Two crossed a-plates as an alternative to a negative c-plate," in SID Int. Symp. Dig. Tech. Papers, Long Beach, CA, May 2000, vol. XXXI, pp. 838-841.

[81] H. C. Huang, B. L. Zhang, H. S. Kwok, P. W. Cheng, and Y. C. Chen, "Color filter liquid-crystal-on-silicon microdisplays," in SID Int. Symp. Dig. Tech. Papers, Boston, MA, May 2005, vol. XXXVI, pp. 880-883.

[82] R. D. Sterling and W. P. Bleha, "D-ILA technology for electronic cinema," in SID Int. Symp. Dig. Tech. Papers, May 2000, vol. XXXI, pp. 310-313.

[83] J. E. Anderson, J. Gandhi, and J. Erdmann, "Vertically aligned fieldsequential microdisplay," in SID Int. Symp. Dig. Tech. Papers, Boston, MA, May 2002, vol. XXXVI, pp. 958-961.

[84] D. Cuypers, H. De Smet, and A. Van Calster, "Fast switching vertically aligned microdisplays," in Proc. 14th Int. Display Workshops, 2007, pp. 2333-2336.

[85] J. Miseli, "Motion artifacts," in SID Int. Symp. Dig. Tech. Papers, 2004, vol. XXXV, pp. 86-89.

[86] W. Song, X. Li, Y. Zhang, Y. Qi, and X. Yang, "Motion-blur characterization on liquid-crystal displays," J. Soc. Inf. Display, vol. 16, no. 5, pp. 587-593, 2008.

[87] J. Someya and H. Sugiura, "Relationship between MPRT measurement and perceived LCD motion blur," in Proc. 25th Int. Display Res. Conf., Sep. 2005, pp. 78-81.

[88] D. Cuypers, G. Van Doorselaer, J. Van Den Steen, H. De Smet, and A. Van Calster, "Assembly of an XGA $0.9^{\prime \prime} \mathrm{LCOS}$ display using inorganic alignment layers for VAN LC," in Conf. Rec. Int. Display Res. Conf., 2002, pp. 551-554.

[89] D. Cuypers, A. Van Calster, and H. De Smet, "Direct measurement of Vcom voltage in liquid crystal panels," in Proc. 16th Int. Display Workshops, 2009, pp. 1377-1380.

[90] M. Lu, K.-H. Yang, and , IBM Corp., "Flicker-free reflective liquid crystal cell," U.S. Patent 5764 324, Jun. 9, 1997.

[91] G. Melnik, P. Janssen, and G. Cnossen, "Galvanic activity in reflective LCDs," in SID Int. Symp. Dig. Tech. Papers, Long Beach, CA, May 2000, vol. XXXI, pp. 252-255.

[92] D. Cuypers, A. Van Calster, and H. De Smet, "Behaviour of compensation voltage in LCOS panels," in Conf. Rec. Int. Display Res. Conf., 2007, pp. 248-251.

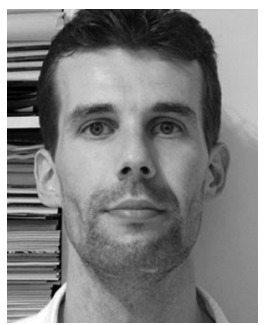

Dieter Cuypers graduated in electromechanical engineering in 1997 and received the Ph.D. degree in electronics engineering in 2005, both from Ghent University, Ghent, Belgium.

$\mathrm{He}$ has been working at the Electronics and Information Systems department since his graduation, doing research for both the university and IMEC. His research is largely situated in the field of visualization technology, specifically microdisplays, but also stretches into other areas like optics and thin-film processing. He is the author or co-author

of over 40 papers.

Dr. Cuypers is a member of SID and SPIE.

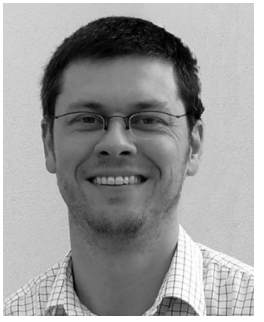

Herbert De Smet graduated in physics engineering in 1988, and received the Ph.D. degree on the subject of integrated drivers for flat panel displays in 1994, both from Ghent University, Ghent, Belgium.

Since 1995, he is working for IMEC, where he is responsible for several European and national research projects concerning displays and microdisplays. Since 2000, he has been a Professor at Ghent University, teaching courses in the field of microsystems, sensors, and actuators.

Dr. De Smet is the secretary of the Mid-Europe chapter of the Society for Information Display, associate editor for the Journal of Information Display and the author or co-author of more than 160 papers.

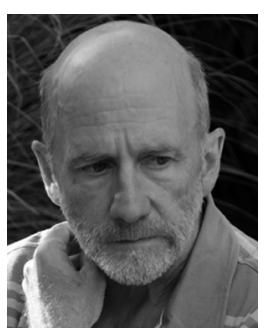

André Van Calster graduated in electronics engineering in 1970, and and received the Ph.D. degree in electronics engineering in 1974, both from Ghent University, Ghent, Belgium, in 1974, both from the same university.

$\mathrm{He}$ joined the department of electronics and information systems (ELIS), where he is now a professor involved in research on semiconductor devices, active-matrix addressing of liquid crystal displays, MCM and interconnection technologies. Currently, he is chairing the research unit CMST (Center for Microsystems Technology), which is affiliated with IMEC. He was the promotor of several PhD's and is the author of over 200 papers.

Dr. Van Calster is a member of IMAPS and SID. He is the treasurer of EURIPIDES (a European Eureka initiative on interconnection technology and MEMS). 